\title{
Irreversible bilayer adsorption of straight semirigid rods on two-dimensional square lattices: Jamming and percolation properties
}

\author{
N. De La Cruz Félix $\odot$ \\ Instituto de Física Aplicada (INFAP), Universidad Nacional de San Luis-CONICET, Ejército de Los Andes 950, D5700HHW \\ San Luis, Argentina \\ and Departamento de Física, Instituto de Física, Facultad de Ciencias, Universidad Autónoma de Santo Domingo, República Dominicana \\ P. M. Centres and A. J. Ramirez-Pastor $\odot^{*}$ \\ Departamento de Física, Instituto de Física Aplicada (INFAP), Universidad Nacional de San Luis-CONICET, Ejército de Los Andes 950, \\ D5700HHW San Luis, Argentina
}

(Received 11 June 2020; accepted 13 July 2020; published 30 July 2020)

\begin{abstract}
Numerical simulations and finite-size scaling analysis have been performed to study the jamming and percolation behavior of straight semirigid rods adsorbed on two-dimensional square lattices. The depositing objects can be adsorbed on the surface forming two layers. The filling of the lattice is carried out following a generalized random sequential adsorption (RSA) mechanism. In each elementary step, (i) a set of $k$ consecutive nearest-neighbor sites (aligned along one of two lattice axes) is randomly chosen and (ii) if each selected site is either empty or occupied by a $k$-mer unit in the first layer, then a new $k$-mer is then deposited onto the lattice. Otherwise, the attempt is rejected. The process starts with an initially empty lattice and continues until the jamming state is reached and no more objects can be deposited due to the absence of empty site clusters of appropriate size and shape. A wide range of values of $k(2 \leqslant k \leqslant 64)$ is investigated. The study of the kinetic properties of the system shows that (1) the jamming coverage $\theta_{j, k}$ is a decreasing function with increasing $k$, with $\theta_{j, k \rightarrow \infty}=0.7299(21)$ the limit value for infinitely long $k$-mers and (2) the jamming exponent $v_{j}$ remains close to 1 , regardless of the size $k$ considered. These findings are discussed in terms of the lattice dimensionality and number of sites available for adsorption. The dependence of the percolation threshold $\theta_{c, k}$ as a function of $k$ is also determined, with $\theta_{c, k}=A+B \exp (-k / C)$, where $A=\theta_{c, k \rightarrow \infty}=0.0457(68)$ is the value of the percolation threshold by infinitely long $k$-mers, $B=0.276(25)$, and $C=14(2)$. This monotonic decreasing behavior is completely different from that observed for the standard problem of straight rods on square lattices, where the percolation threshold shows a nonmonotonic $k$-mer size dependence. The differences between the results obtained from bilayer and monolayer phases are explained on the basis of the transversal overlaps between rods occurring in the bilayer problem. This effect (which we call a "cross-linking effect"), its consequences on the filling kinetics, and its implications in the field of conductivity of composites filled with elongated particles (or fibers) are discussed in detail. Finally, the precise determination of the critical exponents $\nu, \beta$, and $\gamma$ indicates that, although the increasing in the width of the deposited layer drastically affects the behavior of the percolation threshold with $k$ and other critical properties (such as the crossing points of the percolation probability functions), it does not alter the nature of the percolation transition occurring in the system. Accordingly, the bilayer model belongs to the same universality class as two-dimensional standard percolation model.
\end{abstract}

DOI: 10.1103/PhysRevE.102.012153

\section{INTRODUCTION}

An important subject in statistical mechanical is the percolation transition occurring in random sequential adsorption (RSA) models of extended objects on two-dimensional lattices [1-3]. In this type of models, the objects are randomly and irreversibly deposited forming a single monolayer. The final state generated by RSA is a disordered state (known as jamming state), in which no more objects can be deposited due to the absence of free space of appropriate size and shape [2]. Theoretically, the RSA model, introduced by P. J. Flory to treat the interaction between blocks along a linear polymer

\footnotetext{
*antorami@unsl.edu.ar
}

chain [4], has been investigated intensively over past few decades in the context of irreversible processes of adsorption on surfaces [5-9].

The main objective in RSA studies is to find the maximum concentration of extended objects that can be deposited on the surface (jamming coverage). On the other hand, the percolation problem is based on finding the minimum concentration of occupied elements (sites or bonds) for which a spanning cluster extends from one end of the system to the opposite end. This particular value of concentration rate is named percolation threshold. Thus, a competition between percolation and jamming is established.

In the framework of the RSA, percolation of objects of different shapes and sizes have been studied for both lattice and continuum models: linear $k$-mers (objects occupying $k$ 
consecutive sites along a lattice direction) [10-16], flexible polymers [17,18], T-shaped objects and crosses [19], squares [20-23], polydisperse rods [24-26], hard deformed rods [27], hard convex polygonal rods and platelets [28], cylindrical particles [29-31], aligned hard rods [32], etc. These studies have shown that jamming and percolation properties depend strongly on the shape and size of the depositing particles.

In the case of straight rods on discrete lattices, which is the topic of this paper, the percolation and jamming phenomena for linear $k$-mers on two-dimensional (2D) square lattices have been widely studied in the literature [10-16]. In the cited articles, it was shown that (i) the jamming concentration monotonically decreases and tends to $0.660(2)$ as the length of the rods increases [10]. (ii) The percolation threshold is a nonmonotonic function of the size $k$ : It decreases for small rod sizes, goes through a minimum around $k=13$, and finally increases slowly with $k$. For long $k$-mers, the critical threshold tends to 0.615(1) [11-14]. (iii) Any jammed configuration is a percolating state (percolation always occurs before jamming) [15]. And (iv) the problem belongs to the $2 \mathrm{D}$ random percolation universality class regardless of the size of $k$ [16].

The study of these systems in the regime of monolayer offers a basic representation of more complex processes such as adsorption of proteins on solid surfaces [7-9], particles on a biological membrane [33], latex spheres on a silica surface [34,35], etc. Nevertheless, by looking closely at processes such as the adsorption of hydrocarbon chains [36], the crystals growth [37], and the evolution of the surface morphology of pyrolytic graphite [38], the experimental reality suggests the multilayer formation.

In spite of the experimental evidences suggesting that the adsorption process can continue beyond one layer, most of the RSA studies are devoted to deposition at monolayer. However, less attention has received the development of more realistic models considering the formation of a multilayer [39-42]. In a recent paper from our group [43], extensive numerical simulations, supplemented by finite-size scaling theory, were used to study the irreversible multilayer deposition of semirigid $k$-mers on one-dimensional lattices. The jamming threshold $\theta_{n}^{j}(k)$ was reported as a function of the $k$-mer size $(k)$ and the number of deposited layers $(n)$. For a fixed value of $n, \theta_{n}^{j}(k)$ is a decreasing function with the $k$-mer size. In the case of $n=2$ (bilayer problem), $\theta_{n=2}^{j}(k)$ can be fitted by the function $\theta_{n=2}^{j}(k)=0.7708(3)+0.298(3) / k-$ $0.011(7) / k^{2}$, where $0.7708(3)$ represents the limit concentration by infinitely long $k$-mers.

A qualitative similar tendency was observed for the monolayer problem [3,44-47], where $\theta_{n=1}^{j}$ decreases monotonically with $k$ and, for $k \rightarrow \infty$, the jamming threshold tends to Rényi's parking constant $\theta_{n=1}^{j}(k \rightarrow \infty) \rightarrow c_{R} \approx$ 0.7475979202 [47]. However, the possibility of occupying empty sites in the first layer with monomers belonging to $k$-mers that have relaxed from the second layer improves the filling of the lattice and, accordingly, $\theta_{n=1}^{j}(k \rightarrow \infty) \approx$ $0.7475979202<\theta_{n=2}^{j}(k \rightarrow \infty) \approx 0.7708$.

Going back to Ref. [43], an interesting behavior was observed for increasing values of $n$. In this regime, $k$-mers in the $n$th layer adsorb in-registry with the adsorbed $k$-mers in the $(n-1)$ th layer, forming columns of width $k$ separated by valleys of empty sites. As $k$ increases, the separation distance between columns diminishes and the coverage corresponding to the $n$th layer increases with $k$. Thus, for large values of $n$ and long $k$-mers, the jamming coverage tends to approximately 0.71147 . This value is close to the Rényi's parking constant $c_{R} \approx 0.7476$, indicating that (i) the structure of the adsorbed phase in the $n$th layer is similar to that in the monolayer and (ii) the in-registry adsorption governs the deposition kinetics for long $k$-mers. Finally, the critical exponent characterizing the jamming transition was determined, being $v_{j} \approx 2$ as expected for a one-dimensional system.

The investigations in Refs. [39-43] were restricted to onedimensional substrates. Even though interesting aspects of the deposition kinetics and jamming properties were determined, the percolation problem of linear $k$-mers on one-dimensional systems is trivial: The percolating cluster appears only for $k=1$ and full coverage $(\theta=1)$. For $k>1$, the jamming coverage is less than 1 and, consequently, the percolation transition disappears.

In this context, the main objective of the present article is to extend previous studies to 2D systems. For this purpose, the problem of irreversible bilayer adsorption of straight semirigid rods on two-dimensional square lattices has been investigated. We choose the bilayer problem since it is the simplest case of vertical deposition and contains the main properties of the multilayer adsorption. Then, by using extensive numerical simulations and finite-size scaling analysis, jamming and percolation thresholds have been obtained as a function of the $k$-mer size. In both cases, a monotonically decreasing trend was observed. The study also includes a complete analysis of critical exponents and universality.

This work is a natural extension of our previous research in the area of monolayer percolation of polyatomic species. The obtained results contrast significantly with the nonmonotonic tendency observed for the percolation threshold as a function of $k$ in the classical RSA of straight rigid $k$-mers on twodimensional square lattices [11-14]. This finding shows that it is of interest and of value to inquire how the multilayer deposition influences the main percolation properties of extended particles. In addition, and as will be discussed at the end of Sec. IV, the results reported here could have potential application in the field of conductivity in composite materials.

The paper is organized as it follows: The model is presented in Sec. II. Jamming and percolation properties are studied in Secs. III and IV, respectively. Finally, the conclusions are drawn in Sec. V.

\section{THE MODEL}

Let us consider a surface represented by a two-dimensional $L \times L$ square lattice. Straight semirigid $k$-mers can be adsorbed on this surface forming two layers. Accordingly, the positions available for adsorption will be indicated by three indices $(i, j, n)$. The pair $(i, j)$ denotes the location in the square lattice ( $x, y$ coordinates) and $n$ is the layer number: $n=1$ for layer 1 and $n=2$ for layer 2 . The filling of the lattice with straight semirigid $k$-mers is carried out following a generalized RSA process [2]. It consists of three steps, namely (i) a set of $k$ consecutive nearest-neighbor sites (aligned along one of two lattice axes) is randomly chosen, and (ii) if each 


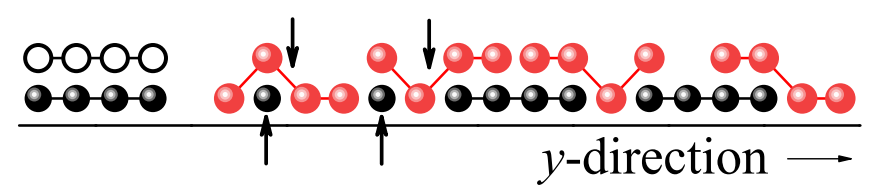

FIG. 1. Schematic representation of tetramers $(k=4)$ deposited along a line in the $y$ direction of the square lattice. As the $k$-mers are semirigid, they can deform to find adjacent empty sites between the first and second layers (but always linear). Black spheres connected by lines represent $k$-mers in the first layer; open spheres connected by lines correspond to $k$-mers in the second layer; and red (gray in grayscale) spheres connected by lines indicate $k$-mers located partially in the first and second layers. The descending arrows in the figure point to $k$-mers adsorbed along the $y$ direction, crossing over transversal $k$-mers previously deposited along the $x$ direction. The units of the tranversal $k$-mers located in the crossing sites are marked by ascending arrows.

selected site is either empty or occupied by a $k$-mer unit in the first layer, then a new $k$-mer is then deposited onto the lattice (see Fig. 1). Otherwise, the attempt is rejected; and (iii) steps (i) and (ii) are repeated until $N k$-mers are deposited in the two layers and the desired concentration $\theta=k N /\left(2 L^{2}\right)$ is reached.

As shown in Fig. 1, each $k$-mer unit occupying a given $(i, j, 2)$ site in the second layer requires the presence of a monomer (belonging to other previously deposited $k$-mer) on the $(i, j, 1)$ site in the first layer. The deposition procedure is performed with periodic boundary conditions in both directions ( $x$ and $y$ axes) and in both layers, so that all sites are statistically equivalent.

The result of the process described above is the formation of a square bilayer of linear semirigid $k$-mers. Then, in addition to the total concentration $\theta$, the partial concentrations $\theta^{l 1}$, $\theta^{l 2}$ can be determined as

$$
\begin{aligned}
\theta^{l 1} & =\frac{\text { number of occupied sites in the first layer }}{L^{2}} \\
& =\frac{N_{1}}{L^{2}}, \\
\theta^{l 2} & =\frac{\text { number of occupied sites in the second layer }}{L^{2}} \\
& =\frac{N_{2}}{L^{2}},
\end{aligned}
$$

and

$$
\theta=\frac{k N}{2 L^{2}}=\frac{N_{1}+N_{2}}{2 L^{2}}=\frac{\theta^{l 1}}{2}+\frac{\theta^{l 2}}{2} .
$$

\section{FILLING KINETICS AND JAMMING COVERAGE}

As it is well known, the irreversible deposition of objects larger than a simple monomer (particle occupying one lattice site) involves the possibility of jamming [2]. Namely, due to the increasing probability of blocking on the lattice by the already randomly deposited objects, the limiting or jamming coverage, $\theta_{j} \equiv \theta(t=\infty)$ is less than that corresponding one for close packing $\left(\theta_{j}<1\right)$. Note that $\theta(t)$ represents the dynamical fraction of lattice sites covered at time $t$ by the deposited objects. Consequently, $\theta$ ranges from 0 to $\theta_{j}$ for objects occupying more than one site. The limiting coverage depends on the structure of the deposited object and the lattice geometry. In this case, our interest is in determining how the jamming coverage is modified when the deposition mechanism leads to the formation of a bilayer structure and the size of the $k$-mer is increased.

To obtain the jamming thresholds in terms of the size $k$, the cumulative frequency $\Phi_{L, k}(\theta)$ that a $L \times L$ lattice reaches a coverage $\theta$ has been calculated taking into account the numerical method applied in Ref. [43]. For this purpose, the range $0 \leqslant \theta \leqslant 1$ is swept, by range index $i$, defining bins of with $2 \times \Delta \theta$. Then we measure the value of the coverage at the position $i, \theta_{i}$, and the corresponding absolute frequency of occurrence $\phi_{L, k}^{\mathrm{abs}}\left(\theta_{i}\right)$. From there we can continue to obtain the frequency of occurrence histogram and to define the cumulative relative frequency, $\Phi_{L, k}(\theta)$. The procedure is the following:

(1) Starting from an initially empty lattice, the $k$-mers are deposited according to the RSA mechanism described in Sec. II. Adsorption of $k$-mers continues until the jamming state is reached.

(2) The absolute frequency is calculated:

$$
\phi_{L, k}^{\mathrm{abs}}\left(\theta_{i}\right)= \begin{cases}+1, & \theta_{i}=\theta_{i} \pm \Delta \theta \\ 0, & \text { for other case }\end{cases}
$$

As mentioned above, the subscript $i$ indicates the central value of the $i$ th bin of the distribution.

(3) The cumulative frequency is calculated as:

$$
\Phi_{L, k}(\theta)=\sum_{i} \phi_{L, k}^{\mathrm{abs}}\left(\theta_{i}\right) .
$$

(4) Finally, $n$ runs of the steps 1-3 are carried out for each lattice size $L$ and each value of $k$, and the cumulative frequency is averaged on the $n$ runs.

In our simulations, $n=2 \times 10^{5}$ and $L / k=64,96,128$, 256,512 , and 640 . Within each series, the ratio $L / k$ was kept constant to avoid spurious results due to the $k$-mer size in comparison with the lattice size. All of this requires extensive computer calculations.

In Fig. 2, the cumulative frequency curves for the different $L / k$ values are shown for the cases $k=2$ and 4 . Independent of the size $k$, the function $\Phi_{L, k}(\theta)$ renders a well-continued curve varying between 0 for an empty lattice to 1 at the jamming condition. For finite systems, the transition is never a step function so we have to observe it by the crossing of the $\Phi_{L, k}(\theta)$ functions assuming that the cases for $L \rightarrow \infty$ will also cross at that point. Then, based on the cumulative frequency functions for various $L / k$ (see Fig. 2), we look for the interval where the curves cross each other. The center of this interval represents the jamming threshold $\theta_{j, k}$ and the width of the interval is the error in the determination of $\theta_{j, k}$. In the figure, this interval is $(0.93194,0.93196)$ for the case $k=2$ and $(0.84374,0.84376)$ for the case $k=4$. Accordingly, $\theta_{j, k=2}=0.93195(1)$ and $\theta_{j, k=4}=0.84375(1)$.

The jamming coverage was obtained for $k$ ranging between 2 and 64 (solid squares). The cases corresponding to $k=32, k=48$, and $k=64$ were calculated for $L / k=64$, $L / k=96$, and $L / k=128$, with an effort reaching almost the limits of our computational capabilities. The results are shown in Fig. 3(a). The curve decreases monotonically as the size $k$ increases. As it can be observed, this behavior is 


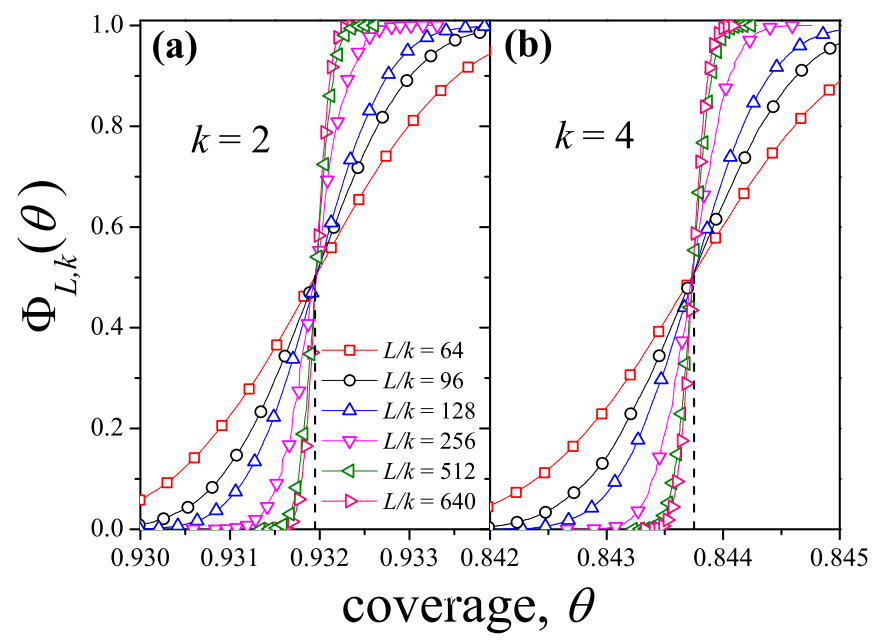

FIG. 2. Cumulative frequency $\Phi_{L, k}(\theta)$ for $k=2$ (a), $k=4$ (b) and different values of $L / k: L / k=64$, squares; $L / k=96$, circles; $L / k=128$, up-triangles; $L / k=256$, down-triangles; $L / k=$ 512 , left-triangles; and $L / k=640$, right-triangles. Vertical dashed lines denote the jamming thresholds in the thermodynamic limit.

also accompanied by a decreasing in the partial concentrations corresponding to the jamming state $\theta_{j, k}^{l 1}$ (layer 1 , open squares) and $\theta_{j, k}^{l 2}$ (layer 2 , solid half squares). As it is expected, $\theta_{j, k}=$ $\left(\theta_{j, k}^{l 1}+\theta_{j, k}^{l 2}\right) / 2$.

As it is standard in the literature $[10,48]$, the simulation data can be fitted to the function $\theta_{j, k}=A+B / k+C / k^{2}(k \geqslant$ 8). In the case of Fig. 3(a),

$$
\theta_{j, k}=0.7299(21)-\frac{0.062(81)}{k}+\frac{3.54(55)}{k^{2}} .
$$

The results from Eq. (6) are included in Fig. 3(a) (solid line). The value of $A$ represents the limit concentration by infinitely long $k$-mers. In this case, $\theta_{j, k \rightarrow \infty}=0.7299(21)$.

In Fig. 3(b), the main curve in part (a), $\theta_{j, k}$ versus $k$, is compared with data of jamming coverage as a function of size $k$ corresponding to (i) irreversible monolayer adsorption of straight rigid $k$-mers on one-dimensional lattices (open circles, Ref. [3]), (ii) irreversible bilayer adsorption of straight semirigid $k$-mers on one-dimensional lattices (solid circles, Ref. [43]), (iii) irreversible monolayer adsorption of straight rigid $k$-mers on 2D square lattices (open squares, Refs. [10,49]), and (iv) irreversible monolayer adsorption of straight rigid $k$-mers on 3D simple cubic lattices (solid spheres, Ref. [50]).

Two main conclusions can be drawn from Fig. 3(b). First, the curve of $\theta_{j, k}$ for the 2D square bilayer tends asymptotically to a limit value $\theta_{j, k \rightarrow \infty}=0.7299(21)$, which is lower than that observed for the one-dimensional bilayer $\theta_{j, k \rightarrow \infty}=0.7708(3)$ [43]. A similar behavior has already been observed for the monolayer problem, where the limiting coverage for infinitely long $k$-mers diminishes with lattice connectivity $c$. Thus, $\theta_{j, k \rightarrow \infty}=0.4045$ (19) (3D simple cubic lattice, $c=6$ ) [50], $\theta_{j, k \rightarrow \infty}=0.660(2)$ (2D square lattice, $\left.c=4\right)[10,49]$, and $\theta_{j, k \rightarrow \infty} \approx 0.7475979$ (1D lattice, $c=2$ ) [3].

Second, the jamming curve obtained in this work for irreversible bilayer adsorption of straight semirigid $k$-mers on 2D square lattices (solid squares) remains above the curve

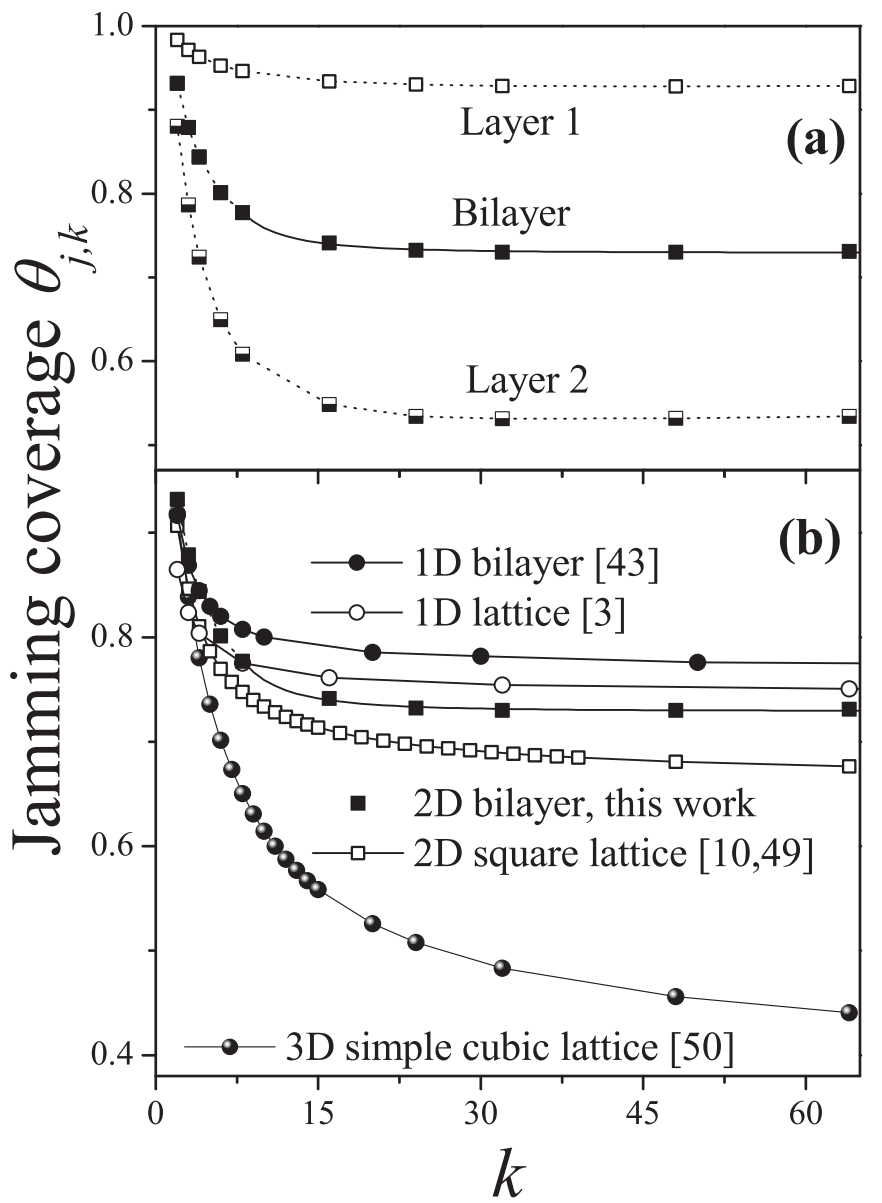

FIG. 3. (a) Jamming coverage $\theta_{j, k}$ as a function of $k$ for irreversible bilayer adsorption of straight semirigid $k$-mers on square lattices. Solid squares represent simulation results (the size of the points is larger than the corresponding error bars). The solid line corresponds to the fitting function as discussed in the text [see Eq. (6)]. The partial concentrations corresponding to the jamming state $\theta_{j, k}^{l 1}$ (layer 1 , open squares) and $\theta_{j, k}^{l 2}$ (layer 2, half solid squares) are included in the figure. (b) The main curve in part (a), $\theta_{j, k}$ versus $k$, is shown in comparison with the data of jamming coverage as a function of size $k$ corresponding to (i) irreversible monolayer adsorption of straight rigid $k$-mers on one-dimensional lattices (open circles, Ref. [3]); (ii) irreversible bilayer adsorption of straight semirigid $k$-mers on one-dimensional lattices (solid circles, Ref. [43]); (iii) irreversible monolayer adsorption of straight rigid $k$-mers on 2D square lattices (open squares, Refs. [10,49]); and (iv) irreversible monolayer adsorption of straight rigid $k$-mers on $3 \mathrm{D}$ simple cubic lattices (solid spheres, Ref. [50]).

corresponding to the monolayer problem (open squares) in all range of $k: \theta_{j, k}^{\text {bilayer }}>\theta_{j, k}^{\text {monolayer }}, k$ ranging between 2 and $\infty$. The same behavior was observed for the one-dimensional problem [open and solid circles in Fig. 3(b)] [43]. This finding can be explained by analyzing the filling process of the bilayer: In a first stage, the depositing particles preferentially occupy the first layer leaving empty sites. These empty sites, which could not be filled in the standard monolayer problem due to the absence of free space of appropriate size and shape, are occupied in the bilayer problem by monomers belonging to $k$-mers that have relaxed from the second layer. The result 
of this process is simple: (i) The limit coverage of the first layer is close to $1\left(\theta_{j, k \rightarrow \infty}^{l 1} \approx 0.92\right)$, and (ii) the total coverage is higher than that corresponding to the monolayer problem in all range of $k$.

In order to complete the jamming study, the critical exponent $v_{j}$ of the jamming transition was obtained. For this purpose, it is useful to fit the quantity $\Phi_{L, k}(\theta)$ by the error function because $d \Phi_{L, k}(\theta) / d \theta$ is expected to behave approximately like the Gaussian distribution. We use the term approximately because the behavior of $d \Phi_{L, k}(\theta) / d \theta$ is known not to be a Gaussian in all range of coverage [51,52]. However, this quantity is approximately Gaussian near the peak, and fitting with a Gaussian function is a good approximation for the purpose of locating its maximum. Thus,

$$
\frac{d \Phi_{L, k}(\theta)}{d \theta}=\frac{1}{\sqrt{2 \pi} \Delta_{L, k}} \exp \left\{-\frac{1}{2}\left[\frac{\theta-\theta_{j, k}(L)}{\Delta_{L, k}}\right]^{2}\right\},
$$

where $\theta_{j, k}(L)$ is the concentration at which the slope of $\Phi_{L, k}(\theta)$ is maximum and $\Delta_{L, k}$ is the standard deviation from $\theta_{j, k}(L)$.

According to the finite-size scaling theory, it is expected that

$$
\left(\frac{d \Phi_{L, k}}{d \theta}\right)_{\max } \propto L^{1 / v_{j}}
$$

and

$$
\Delta_{L, k} \propto L^{-1 / v_{j}}
$$

Figure 4(a) shows $\left(d \Phi_{L, k} / d \theta\right)_{\max }$ and $\Delta_{L, k}$ (inset) as a function of $L / k$ for $k=2$ (circles) and $k=4$ (squares). Following the scheme given in Eqs. (7)-(9), the value of $v_{j}$ can be obtained from the slopes of the curves (the line is a linear fit of the points). In both cases (main figure and inset), the critical exponent obtained from the slope of the curves is close to 1 . The procedure was repeated for different values of $k$. In all the cases, the values obtained for $v_{j}$ (1) remain close to 1 and (2) coincide, within the numerical errors, with the values previously reported in other $2 \mathrm{D}$ monolayer systems [20,23,52-54].

As demonstrated for monolayer deposition [54], $\left(\Delta_{L, k}\right)^{-1}$, $\left(\frac{d \Phi_{L, k}}{d \theta}\right)_{\max } \propto M^{1 / 2}$, where $M$ is the number of elements (sites or nodes) that form the lattice. The results in Fig. 4(a) allow us to generalize this scheme for bilayer deposition. In fact, as discussed in Sec. II, $M=2 L^{2}$ for the bilayer problem. Then,

$$
\left(\frac{d \Phi_{L, k}}{d \theta}\right)_{\max },\left(\Delta_{L, k}\right)^{-1} \propto M^{1 / 2}=\left(2 L^{2}\right)^{1 / 2} \propto L,
$$

and accordingly, a value of $v_{j}=1$ is expected.

As shown in Figs. 4(b), the properties of $\Phi_{L, k}(\theta)$ are identical to those of $R_{L, k}^{X}(\theta)$ in standard percolation transitions (this probability will be discussed in details in the next section). Namely, $R_{L, k}^{X}(\theta)$ obeys the same scaling relation in Eqs. (8) and (9), and the intersection of the curves of $R_{L, k}^{X}(\theta)$ for different system sizes can be used to determine the critical point that characterizes the percolation transition occurring in the system. Then, based on these features, we propose the following scaling behavior at criticality for the cumulative
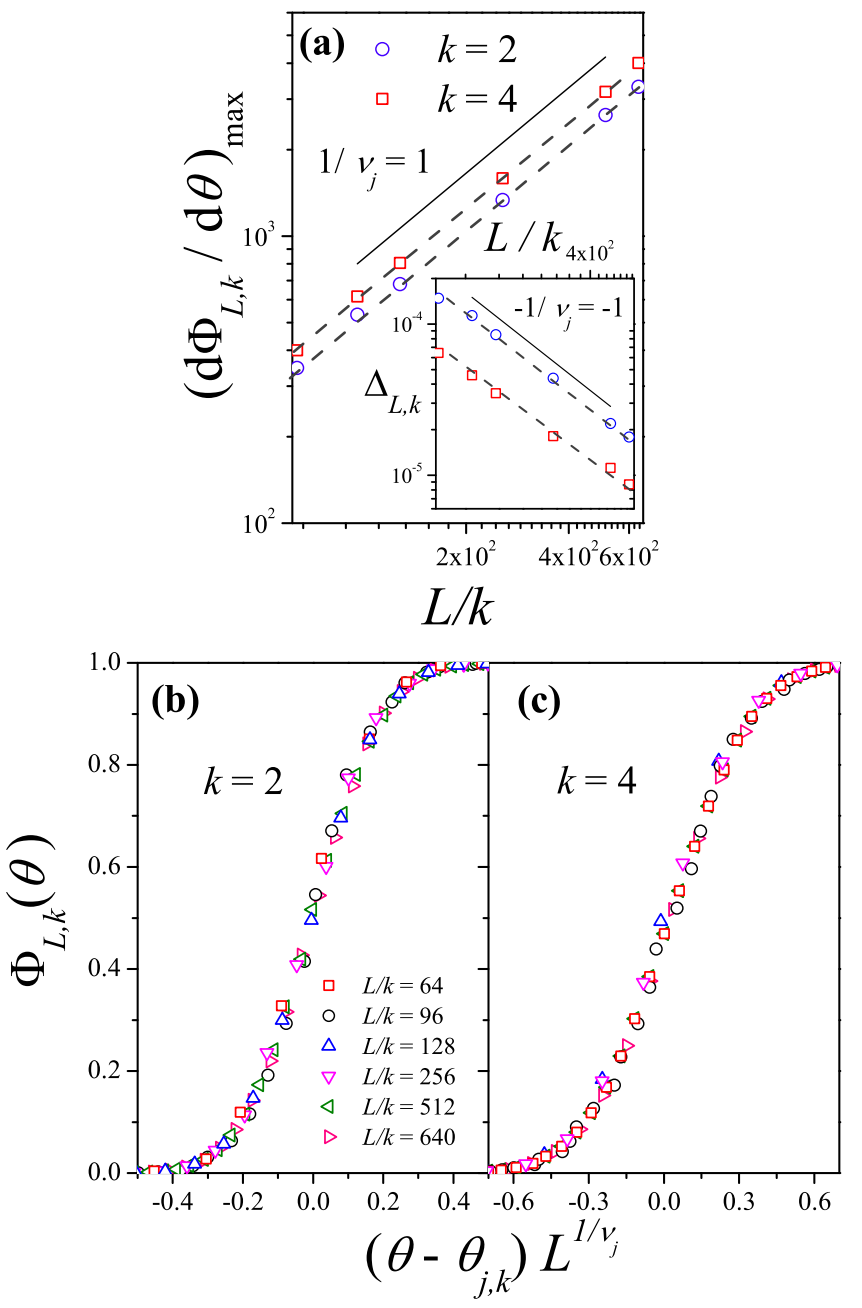

FIG. 4. (a) Log-log plots of $\left[d \Phi_{L, k}(\theta) / d \theta\right]_{\max }$ and $\Delta_{L, k}$ (inset) as a function of $L / k$ for the cases shown in Fig. 2: $k=2$, circles; and $k=4$, squares. According to Eq. (8) the slope of each line corresponds to $1 / v_{j}$ [or to $-1 / v_{j}$ in the case of Eq. (9)]. (b) Data collapse of the cumulative frequency, $\Phi_{L, k}(\theta)$ versus $\left(\theta-\theta_{j, k}\right) L^{1 / v_{j}}$ [Eq. (11)] for $k=2$. The curves were obtained using $\theta_{j, k=2}=0.93195$ (1) (see Fig. 2) and $v_{j}=1$. (c) Same as in (b) but for $k=4$. In this case, the curves were obtained using $\theta_{j, k=4}=0.84375(1)$ (see Fig. 2) and $v_{j}=1$.

frequency:

$$
\Phi_{L, k}(\theta)=\overline{\Phi_{k}}\left[\left(\theta-\theta_{j, k}\right) L^{1 / v_{j}}\right],
$$

where $\overline{\Phi_{k}}$ is the corresponding scaling function.

The scaling tendency in Eq. (11) has been tested by plotting $\Phi_{L, k}(\theta)$ versus $\left(\theta-\theta_{j, k}\right) L^{1 / v_{j}}$ and looking for data collapsing. As an example, Fig. 4(b) shows the obtained results for $k=2$ and $k=4$. Using the values of $\theta_{j, k=2}=0.93195(1), \theta_{j, k=4}=$ $0.84375(1)$ (see Fig. 2) and $v_{j}=1$, the curves present an excellent scaling collapse. This data collapse study allows for consistency check of the value $v_{j}=1$ calculated in Fig. 4(a).

\section{PERCOLATION PROPERTIES}

With the space of the parameter $\theta$ determined, the percolation properties of the system will be studied in this section. 
The percolation theory describes the behavior of connected clusters in a given lattice. In the case of site-lattice models, which is the topic of this paper, a cluster is a group of occupied sites in such a way that each one of them has at least one occupied nearest-neighbor site. For a precise value of concentration, a cluster of nearest-neighbor sites extends from one side to the opposite side of the system. This particular value of the coverage degree is named percolation threshold and determines a well-defined second order transition in the system. Here we are interested in determining (i) the percolation threshold as a function of the size of the semirigid rods $\theta_{c, k}$ and (ii) the universality class of the percolation transition. These results will allow us to discuss the interplay between jamming and percolation phenomena.

\section{A. Calculation method and percolation thresholds}

The finite-scaling theory gives us the basis to determine the percolation threshold and the critical exponents of a system with a reasonable accuracy. For this purpose, the probability $R=R_{L, k}^{X}(\theta)$ that a lattice of linear size $L$ percolates at concentration $\theta$ of occupied sites by $k$-mers can be defined [55]:

(i) $R_{L, k}^{R}(\theta)$ : the probability of finding a rightward percolating cluster, along the $x$ direction,

(ii) $R_{L, k}^{D}(\theta)$ : the probability of finding a downward percolating cluster, along the $y$ direction,

(iii) $R_{L, k}^{U}(\theta)$ : the probability of finding a cluster which percolates on any direction,

(iv) $R_{L, k}^{I}(\theta)$ : the probability of finding a cluster which percolates in both (mutually perpendicular) directions, and

(v) $R_{L, k}^{A}(\theta)=\frac{1}{2}\left[R_{L, k}^{U}(\theta)+R_{L, k}^{I}(\theta)\right]$.

The percolation properties can be estimated from the curves of $R_{L, k}^{X}(\theta)$ for the different values of $L$ and $k$. In the simulations, each run consists of the following steps:

(a) the construction of a square bilayer of side $L$ with a coverage $\theta$, according to the deposition scheme introduced in Sec. II;

(b) the cluster analysis by using the Hoshen and Kopelman algorithm [56], with the following connectivity criterion: Each position $(i, j, 1)$ in the first layer has four nearest-neighbor positions in the first layer $[(i-1, j, 1),(i+1, j, 1),(i, j-$ $1,1)$, and $(i, j+1,1)]$ and one nearest-neighbor position in the second layer $[(i, j, 2)]$. In the same way, each position $(i, j, 2)$ in the second layer has four nearest-neighbors positions in the second layer $[(i-1, j, 2),(i+1, j, 2),(i, j-$ $1,2)$, and $(i, j+1,2)]$ and one nearest-neighbor position in the first layer $[(i, j, 1)]$; and

(c) the determination of the largest cluster $S_{L}$, and, finally, the existence of a percolating island. In steps (b) and (c), open boundary conditions are implemented.

A total of $m$ independent runs of such three steps procedure were carried out for each lattice size $L$ and $k$-mer size $k$. Then, the probabilities have been calculated as $R_{L, k}^{X}(\theta)=$ $m^{X} / m$, where $m^{X}$ indicates the number of percolating samples according to the criterion $X(=R, D, U, I, A)$.

In addition, the percolation order parameter and its corresponding susceptibility $\chi$ have been obtained from the largest cluster [57-59],

$$
P=\frac{\left\langle S_{L}\right\rangle}{2 L^{2}},
$$

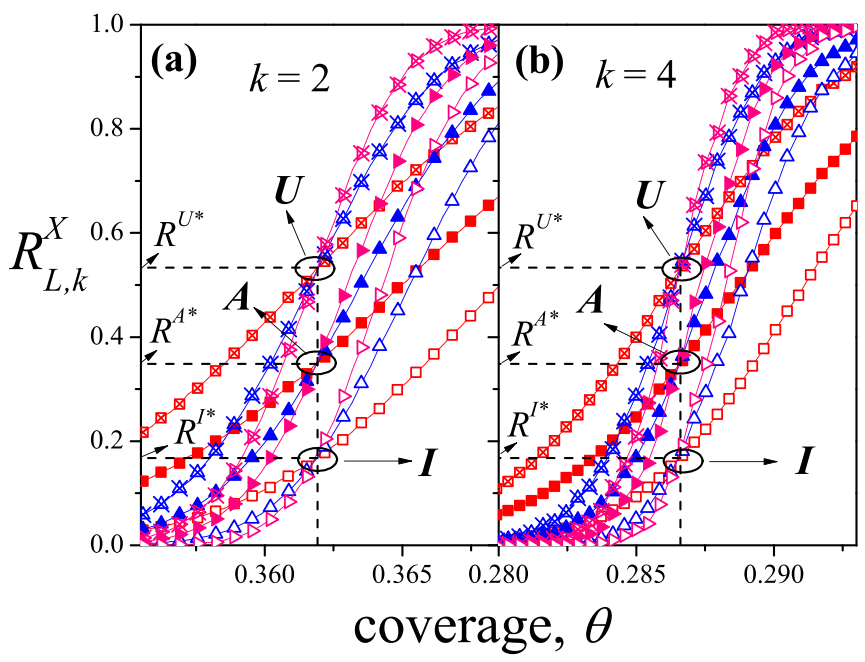

FIG. 5. Fraction of percolating lattices $R_{L, k}^{X}(\theta)$ as a function of the concentration $\theta$ for $k=2$ (a), $k=4$ (b), and three different lattice sizes: $L / k=128$, squares; $L / k=320$, up-triangles; and $L / k=512$, right-triangles. Solid, open and crossed symbols represent data for $A, I$, and $U$ criteria, respectively. The statistical errors are smaller than the symbol sizes. Horizontal dashed lines show the $R^{X^{*}}$ crossing points. Vertical dashed lines denote the percolation threshold in the thermodynamic limit $L \rightarrow \infty$.

where $<. .>$ means an average over simulation runs, and

$$
\chi=\frac{\left[\left\langle S_{L}^{2}\right\rangle-\left\langle S_{L}\right\rangle^{2}\right]}{2 L^{2}},
$$

respectively.

In our percolation simulations, we used $m=2 \times 10^{5}$ independent random samples. In addition, for each value of $k$, the effect of finite size was investigated by examining square lattices with $L / k=128,256,320,384,448,512$. As it can be appreciated, this represents extensive calculations from the computational point of view. From this analysis, finite-scaling theory can be used to determine the percolation threshold and the critical exponents with reasonable accuracy.

The probability curves $R_{L, k}^{X}(\theta)$ are shown in Fig. 5 for $k=$ 2 [Fig. 5(a)] and $k=4$ [Fig. 5(b)]. Solid, open, and crossed symbols represent data for $A, I$, and $U$ criteria, respectively. As mentioned above, the simulations were performed for lattice sizes ranging between $L / k=128$ and $L / k=512$. For simplicity, three sizes are shown in the figure: $L / k=128$ (squares), $L / k=320$ (up-triangles), and $L / k=512$ (righttriangles). From Fig. 5(a) and 5(b), it is observed that the $y$ axis values of the crossing points $\left(R^{X^{*}}\right)$ depend on the criterion $X$ used: $R^{A^{*}} \approx 0.35, R^{I^{*}} \approx 0.17$, and $R^{U^{*}} \approx 0.53$.

The critical crossing points play an important role in the scaling theory, giving a preliminary indication of the universality class of the transition. From this perspective, two main conclusions can be extracted from the results in Fig. 5. First, the crossing points do not modify their numerical value for the different $k$ sizes. This finding is indicative that the universality class of the percolation transition involved in the problem is conserved no matter the values of $k$.

Second, the $R^{X^{*}}$ values for the bilayer problem are different to the corresponding exact values for standard percolation: 
A criterion, $1 / 2$ [60,61], I criterion, $0.32212045 \ldots$ [61,62], and $U$ criterion, $0.67788954 \ldots[61,62]$. This result could be taken as a first indication of a nonuniversal behavior of the system. However, as pointed out by Selke et al. [63,64], the measure of $R^{X^{*}}$ may depend on various details of the model which do not affect the universality class: boundary conditions, lattice shape, anisotropy of the system, etc. In this case, the differences can be attributed to the possibility of formation of a bilayer. Consequently, more research is required to determine the universality class of the percolation transition. This will be carried out in Sec. IV B.

In addition, the intersection points are located at very well defined values in the $\theta$ axes, allowing a preliminary determination of the critical percolation threshold for each $k$. However, the transition is never sharp for finite systems. Accordingly, each intersection point is not a unique point and shows a slight shift with changes of the lattice size $L$. As we will show next, the scaling theory offers more accurate ways to determinate the percolation thresholds.

One of these ways, which will be used here, allows us to estimate accurately the percolation threshold from the maximum of the curves of $R_{L, k}^{X}(\theta)[1,55,57]$. For this, it is convenient to express the different curves as functions of continuous values of $\theta$. Then, as in the case of the jamming probability, $d R_{L, k}^{X} / d \theta$ can be fitted by the Gaussian function:

$$
\frac{d R_{L, k}^{X}}{d \theta}=\frac{1}{\sqrt{2 \pi} \Delta_{L, k}^{X}} \exp \left\{-\frac{1}{2}\left[\frac{\theta-\theta_{c, k}^{X}(L)}{\Delta_{L, k}^{X}}\right]^{2}\right\},
$$

where $\theta_{c, k}^{X}(L)$ is the concentration at which the slope of $d R_{L, k}^{X} / d \theta$ is the largest and $\Delta_{L, k}^{X}$ is the standard deviation from $\theta_{L, k}^{X}(L)$.

Once the values of $\theta_{c, k}^{X}(L)$ were obtained for all lattice sizes, the percolation thresholds were obtained by scaling analysis [1]. In this way, the following relationship is

$$
\theta_{c, k}^{X}(L)=\theta_{c, k}^{X}(\infty)-A^{X} L^{-1 / v}
$$

where $A^{X}$ is a nonuniversal constant and $v$ is the critical exponent of the correlation length which has been taken as $4 / 3$ for the present analysis, since, as it will be shown below, our model belongs to the same universality class as random percolation [1].

Figure 6 shows the extrapolation toward the thermodynamic limit of $\theta_{c, k}^{X}(L)(X=I, U, A$ and $k=2,4)$ according to Eq. (15). Then, the final values of $\theta_{c, k}^{X}(\infty)$ are given as $\theta_{c, k} \pm \delta_{k}$, where $\delta_{k}=\max \left(\left|\theta_{c, k}^{U}-\theta_{c, k}^{A}\right|,\left|\theta_{c, k}^{I}-\theta_{c, k}^{A}\right|\right)$. The values obtained in Fig. 6 were $\theta_{c, k=2}(\infty)=0.3619(2)$ and $\theta_{c, k=4}(\infty)=0.2866(2)$. For the rest of the paper, we will denote the percolation threshold for each size $k$ by $\theta_{c, k}$ [for simplicity we will drop the symbol " $(\infty)$ "].

The procedure of Fig. 6 was repeated for $k$ ranging between 2 and 64, and the results are shown in Fig. 7(a) (solid squares). The corresponding values of $\theta_{c, k}^{l 1}$ and $\theta_{c, k}^{l 2}$ are plotted in open squares and half solid squares, respectively. The points corresponding to $k=32,48$, and 64 were calculated for three relatively small values of $L / k=(96,128,192)$. The percolation threshold decreases on increasing $k$. At the beginning, for small values of $k$, the curve rapidly decreases.

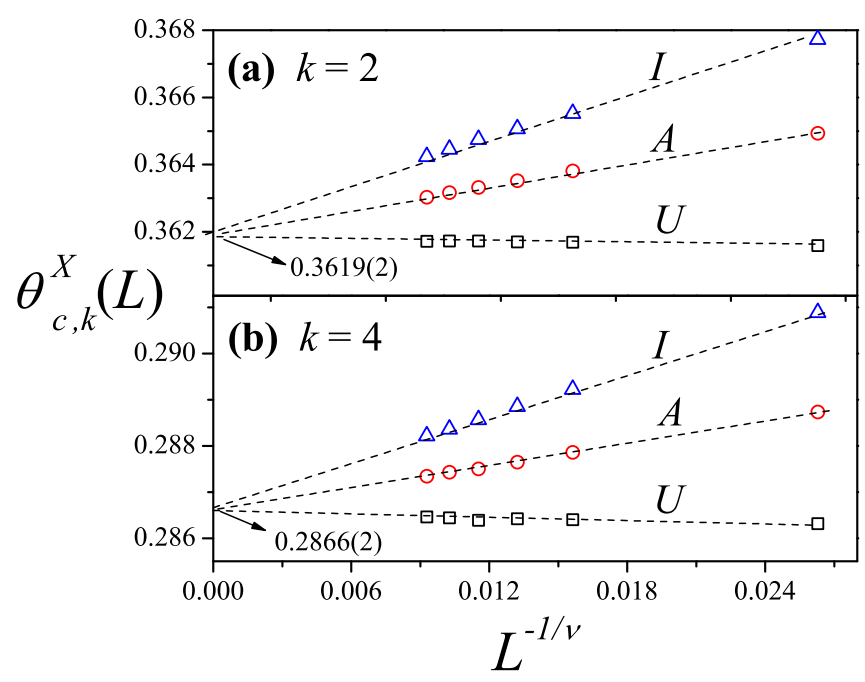

FIG. 6. Extrapolation of the percolation threshold for an $L$ lattice $\theta_{c, k}^{X}(L)(X=\{I, U, A\})$ toward the thermodynamic limit according to the theoretical prediction given by Eq. (15). Triangles, circles, and squares denote the values of $\theta_{c, k}^{X}(L)$ obtained by using the criteria $I$, $A$, and $U$, respectively. Two values of $k$ are presented: (a) $k=2$ and (b) $k=4$. The bar error in each measurement is smaller than the size of the corresponding symbol.

However, it flatten out for larger values of $k$ and finally asymptotically converges toward a definite value as $k \rightarrow \infty$. This limit point can be obtained by fitting the simulation data to the function $\theta_{c, k}=A+B \exp (-k / C)(A, B$, and $C$ being the fitting parameters), as proposed in Ref. [16] for the problem of bond percolation of linear segments of length $k$. In this case, it is found that $A=\theta_{c, k \rightarrow \infty}=0.0457(68), B=0.276(25)$, and $C=14(2)$. The fitting curve is shown in Fig. 7(a) (solid line).

In Fig. 7(b), the main curve in Fig. 7(a) (solid squares) is compared with the data of percolation threshold as a function of size $k$ corresponding to (i) irreversible monolayer adsorption of straight rigid $k$-mers on 2D square lattices (open squares, Ref. [14]) and (ii) irreversible monolayer adsorption of straight rigid $k$-mers on 3D simple cubic lattices (solid spheres, Ref. [65]). As is expected, the bilayer percolation curve is located between the $2 \mathrm{D}$ monolayer curve and the $3 \mathrm{D}$ curve.

With respect to the behavior of the curve of $\theta_{c, k}$ reported in this work, there are two aspects that merit to be commented. On the one hand, the decreasing tendency of the bilayer percolation curve is qualitatively similar to the one for irreversible monolayer adsorption of straight rigid $k$-mers on 3D simple cubic lattices [65]. On the other hand, this monotonous behavior contrasts sharply with that observed for the 2D monolayer problem [14], where the percolation threshold shows a nonmonotonic $k$-mer size dependence. Namely, the percolation threshold decreasing for small particle sizes, going through a minimum at $k \approx 13$, and finally slowly increasing as $k$ increases. For long $k$-mers $(k \rightarrow \infty)$, the $\theta_{c, k}$ curve tends to a saturation value around 0.615 [14].

The notorious differences between the decreasing tendency of the percolation curve $\left(\theta_{c, k}\right.$ versus $\left.k\right)$ for the 2D bilayer problem [solid squares in Fig. 7(b)] and the nonmonotonous 


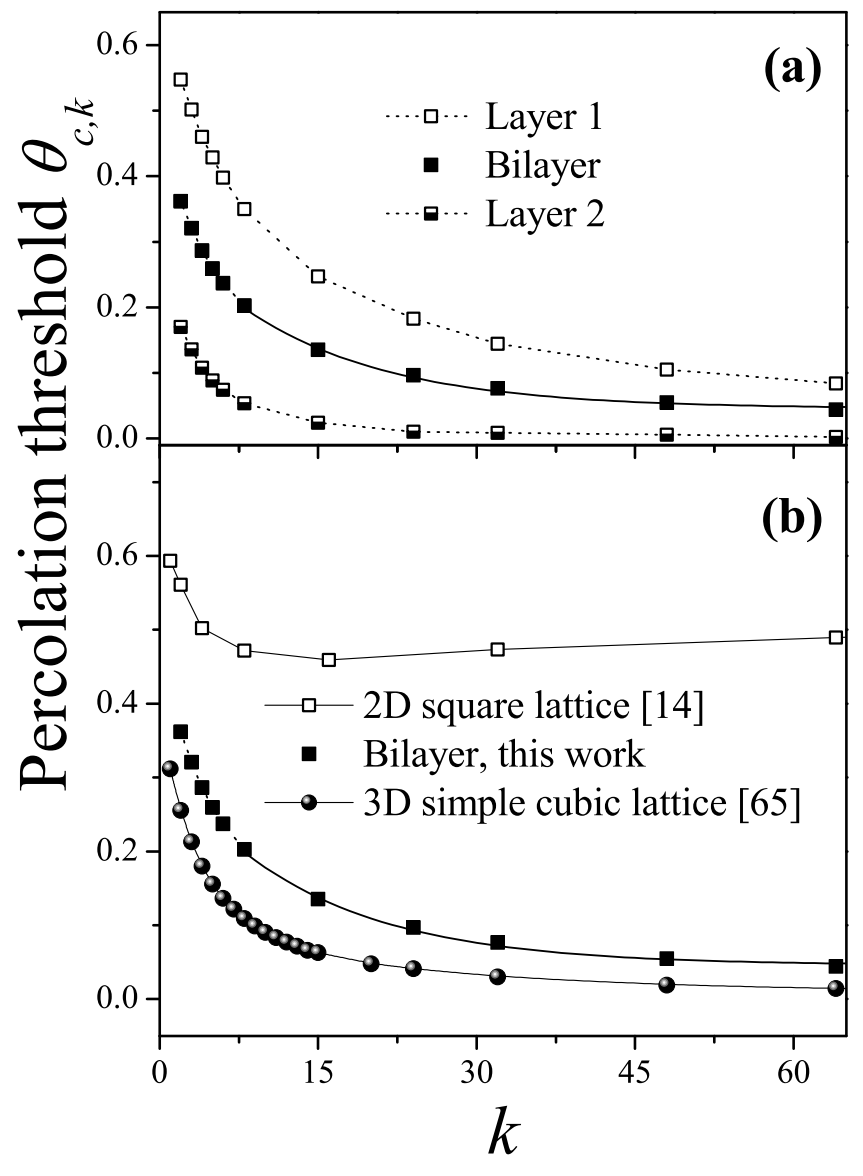

FIG. 7. (a) Percolation threshold $\theta_{c, k}$ as a function of $k$ for irreversible bilayer adsorption of straight semirigid $k$-mers on square lattices. Solid squares represent simulation results (the size of the points is larger than the corresponding error bars). The solid line corresponds to the fitting function $\theta_{c, k}=A+B \exp (-k / C)$ as discussed in the text. The partial concentrations corresponding to the percolation state $\theta_{c, k}^{l 1}$ (layer 1, open squares) and $\theta_{c, k}^{l 2}$ (layer 2, half solid squares) are included in the figure. (b) The main curve in part (a), $\theta_{c, k}$ versus $k$, is shown in comparison with the data of percolation threshold as a function of size $k$ corresponding to (i) irreversible monolayer adsorption of straight rigid $k$-mers on 2D square lattices (open squares, Ref. [14]) and (ii) irreversible monolayer adsorption of straight rigid $k$-mers on 3D simple cubic lattices (solid spheres, Ref. [65]).

dependence of the percolation threshold on the size $k$ observed for the 2D monolayer problem [open squares in Fig. 7(b)] can be understood by looking at the role of those $k$-mers in the second layer, which are placed on transversal rods previously deposited in the first layer. Illustrative examples of the occurrence this "cross-linking effect" [66] are shown in Fig. 1. There the $k$-mers pointed by descending arrows were adsorbed along the $y$ direction, crossing over transversal $k$-mers previously deposited along the $x$ direction. The units of the tranversal $k$-mers located in the crossing sites are marked by ascending arrows.

The cross-linking rule described above is not permitted in the 2D monolayer problem, where overlaps between rods are not allowed. In this case, as established by several authors

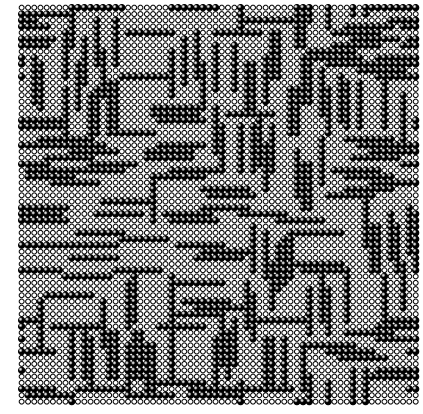

(a)

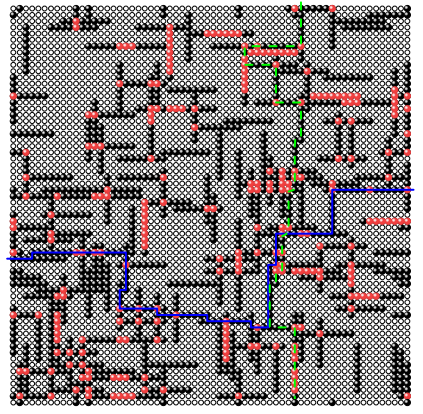

(b)

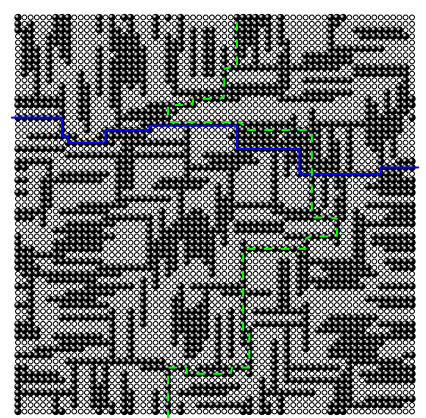

(c)

FIG. 8. (a) Typical configuration of $k$-mers of length $k=8$ irreversibly deposited on a $64 \times 64$ square lattice at the monolayer regime. The number of deposited $k$-mers is $N=210$ and the corresponding coverage is $\theta=k N / L^{2} \approx 0.4102$. Black spheres and open circles represent $k$-mer units and empty sites, respectively. (b) Same as in (a) for the bilayer problem. In this case, $\theta=$ $k N /\left(2 L^{2}\right) \approx 0.2051$ and the corresponding partial concentrations are $\theta^{l 1}=k N_{1} / L^{2} \approx 0.3486$ and $\theta^{l 2}=k N_{2} / L^{2} \approx 0.0616$. Black spheres, red spheres (gray spheres in grayscale), and open circles represent $k$-mer units in the first layer, $k$-mer units in the second layer and empty sites, respectively. Dashed green (solid blue) lines indicate percolation paths connecting top and bottom (left and right) sides of the lattice. (c) Same as in (a) for $N=242$ and, consequently, $\theta=k N / L^{2} \approx 0.4727$. As in (b), dashed green (solid blue) lines indicate percolation paths connecting top and bottom (left and right) sides of the lattice.

$[11,13]$, the formation of vertical and horizontal blocks of parallel $k$-mers reverses the initial decrease in $\theta_{c, k}$, leading to the appearance of a minimum in the curve of percolation threshold as a function of the size $k$. It is well known that for ideal blocks $(k \times k$ squares $)$, the percolation concentration increases with $k$, and above certain critical value of $k$ no percolation is observed $[20,23,52]$. The apparition of the crosslinking effect in the bilayer problem changes drastically the filling kinetics, strongly suppressing the formation of domains or islands of parallel $k$-mers, and, consequently, leading to the disappearance of the minimum observed in the classical percolation problem of straight rigid $k$-mers on 2D square lattices. The cross-linking effect also favors connectivity, causing the percolation transition to occur at lower values of coverage. These concepts can be better visualized with the help of the next figure.

Figure 8 shows typical configurations of $k$-mers of length $k=8$ irreversibly deposited on a $64 \times 64$ square lattice at different conditions: (a) monolayer regime, $N=210$ 
and $\theta=k N / L^{2} \approx 0.4102$; (b) bilayer regime, $N=210$ and $\theta^{l 1}=k N_{1} / L^{2} \approx 0.3486, \theta^{l 2}=k N_{2} / L^{2} \approx 0.0616$ and $\theta=$ $k N /\left(2 L^{2}\right) \approx 0.2051$; and (c) monolayer regime, $N=242$ and $\theta=k N / L^{2} \approx 0.4727$. The coverage in Fig. 8(a) is less than that required for percolation. The concentrations in Figs. 8(b) and $8(\mathrm{c})$ are slightly above the corresponding percolation thresholds. The meaning of the symbols is indicated in the figure.

In cases (a) and (b), the same number of $k$-mers has been deposited. However, the resulting configurations are very different. In case (a), an incipient tendency to the formation of domains of parallel $k$-mers is observed. The structure of the adsorbed phase is composed of a large number of isolated clusters, and it does not contain a spanning cluster connecting two opposite sides of the system. In case (b), the cross-linking effect governs the structure of the adsorbed phase [note the large number of crossing sites that can be identified in Fig. 8(b)]. This favors the connection between groups of occupied sites, and avoids the growth of domains with local alignment as observed in case (a). In addition, there are a few isolated islands of nearest-neighbor occupied sites, and a spanning cluster extends from one end of the lattice to the opposite end. Dashed green (solid blue) lines indicate percolation paths connecting top and bottom (left and right) sides of the lattice.

The differences between monolayer and bilayer problems are even more evident when the comparison is made between percolating states. For this purpose, Fig. 8(c) shows a typical percolation configuration for the monolayer problem. There, blocks of parallel $k$-mers dominate the structure of the adsorbed phase, and a higher amount of deposited $k$-mers is required to reach percolation.

The study in Fig. 8 clearly shows that (i) the mechanism of local alignments that governs the nonmonotonic behavior exhibited by the percolation curve for the monolayer problem does not work when the $k$-mers are adsorbed forming a bilayer and (ii) the structure of the adsorbed phase for the bilayer problem is dominated by the cross-linking effect, which favors the connectivity and explains the differences observed between monolayer and bilayer systems.

From the experimental point of view, fiber-reinforced composites have been used increasingly as a structural material, partly due to their high stiffness and strength-to-weight ratio. In this framework, it is important to consider the contribution of the aspect ratio of the fibers to the electrical conductivity of the composites; such a role has been emphasized by various authors [29-31,67-72]. Theoretically, the electric conductive property of polymer matrix composites filled with conductive particles or fibers can be characterized by a percolation transport process. Accordingly, percolation theory is frequently applied to describe the insulator-to-conductor transitions in composite materials made of conductive rods or fibers and an insulating matrix.

In this context, experimental measurements on the critical volume fractions of polypropylene-vapor grown carbon fiber (PP-VGCF) with average aspect ratio of 100 and polystyrenevapor grown carbon fiber (PS-VGCF) with average aspect ratio of 400 were been carried out by Ohtake et al. [70] and Gordeyev et al. [71]. The results obtained for these thin films indicates that the critical volume fraction (or percolation threshold) decreases as the aspect ratio is increased. Motivated by these findings, a 2D continuum model of overlapping rods with a finite width was introduced in Ref. [72]. Based on the presented model and Monte Carlo simulations, the authors reported a monotonically decreasing behavior of the percolation threshold as a function of the aspect ratio. In addition, the predicted theoretical results showed a good agreement with the experimental data in Refs. [70,71]. A similar decreasing behavior was observed from experimental [29] and theoretical studies of silver nanowire-polystyrene composites [29-31].

The dependence of the percolation threshold with the aspect ratio in Refs. [29-31,72] is similar to that presented in Fig. 7 for the 2D bilayer problem. This result provides an important contribution to the understanding of these systems, namely, the inclusion of the cross-linking rule in the model is essential to describe the monotonically decreasing of the percolation threshold curve. In 2D discrete lattice models, the possibility of formation of multilayers allows to incorporate this crucial effect. If the $k$-mers do not cross each other, such as occur in the case of 2D discrete lattice models at monolayer $[14,48]$, then a nonmonotonous behavior should be expected for the percolation threshold as a function of the size $k$.

The considerations given in this section provide one more tool for analysis of experimental data of conductivity in fiberreinforced laminated composites, allowing us to shed light on various interesting questions such as formation (or not) of multilayer, overlapping between the conductive rods, etc. In addition, the confirmation of the theoretical predictions for the minimum reported in monolayers of nonoverlapping rigid rods represents an important challenge for experimentalists.

\section{B. Critical exponents and universality}

In order to investigate the universality of the system, the critical exponents $\nu, \beta$, and $\gamma$ have been calculated. Knowing this set of exponents allows to determine the universality class of our system and understand the related phenomena. This study will also allow us to understand why the values of the crossing points $R^{X^{*}}$ vary for the bilayer problem with respect to the standard percolation problem.

The standard theory of finite size [57] allows for various routes to estimate the critical exponent $v$ from simulation data. One of these methods is from the maximum of the function $d R_{L, k}^{X} / d \theta$ [Eq. (7)],

$$
\left(\frac{d R_{L, k}^{X}}{d \theta}\right) \propto L^{1 / \nu} .
$$

In Fig. 9(a), $\log \left[\left(d R_{L, k}^{X} / d \theta\right)_{\text {max }}\right]$ has been plotted as a function of $\log [L]$ for $k=2$ (circles) and $k=4$ (squares). According to Eq. (16), the slope of each line corresponds to $1 / \nu$. As it can be observed, the slopes of the curves remain constant and close to $v=4 / 3$.

Another alternative way for evaluating $v$ is from the divergence of the root mean square deviation of the percolation threshold observed from their average values, $\Delta_{L, k}^{X}$,

$$
\Delta_{L, k}^{X} \propto L^{-1 / v}
$$




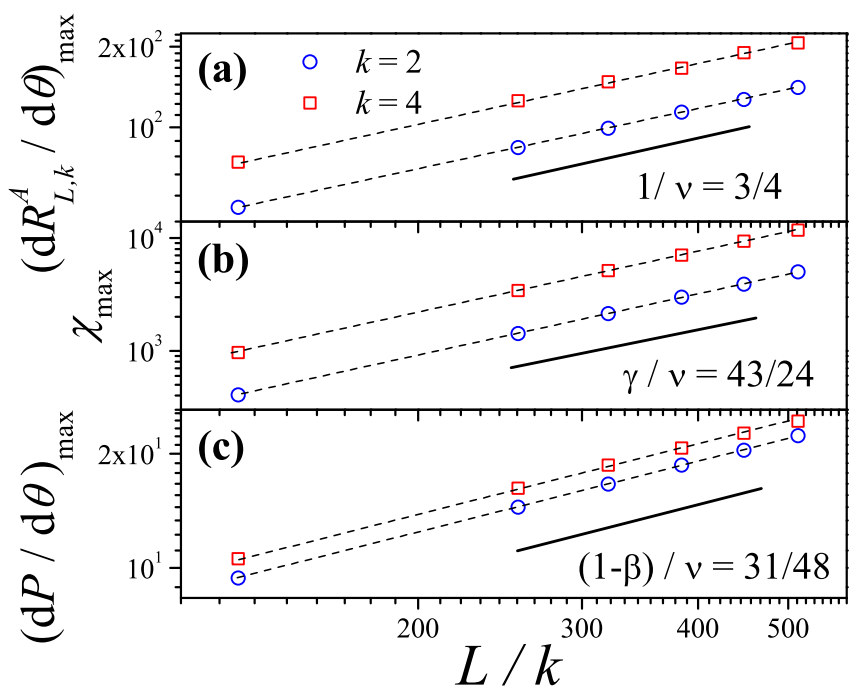

FIG. 9. (a) Maximum of the derivative of the $A$ percolation probability $\left(d R_{L, k}^{A} / d \theta\right)_{\max }$ as a function of $L$ (in a log-log scale) for two different cases: $k=2$ (circles) and $k=4$ (squares). According to Eq. (16) the slope of each line corresponds to $1 / v=3 / 4$. (b) Maximum of the susceptibility $\chi_{\max }$ as a function of $L$ (in a $\log -\log$ scale) for the cases in part (a). The slope of each line corresponds to $\gamma / v=43 / 24$ [Eq. (20)]. (c) Maximum of the derivative of the percolation order parameter $(d P / d \theta)_{\max }$ as a function of $L$ (in a $\log$-log scale) for the same cases reported in part (a). According to Eq. (21), the slope of each line corresponds to $(1-\beta) / v=31 / 48$.

$\Delta_{L, k}^{X}$ was studied as a function of $L$ (data not shown for brevity), and the values obtained for $-1 / v$ were consistent with $v=4 / 3$.

The calculation of $v$ was repeated for each value of $k$ and the $A, I$, and $U$ percolation criteria. In all cases, the obtained results coincide, within numerical errors, with the exact

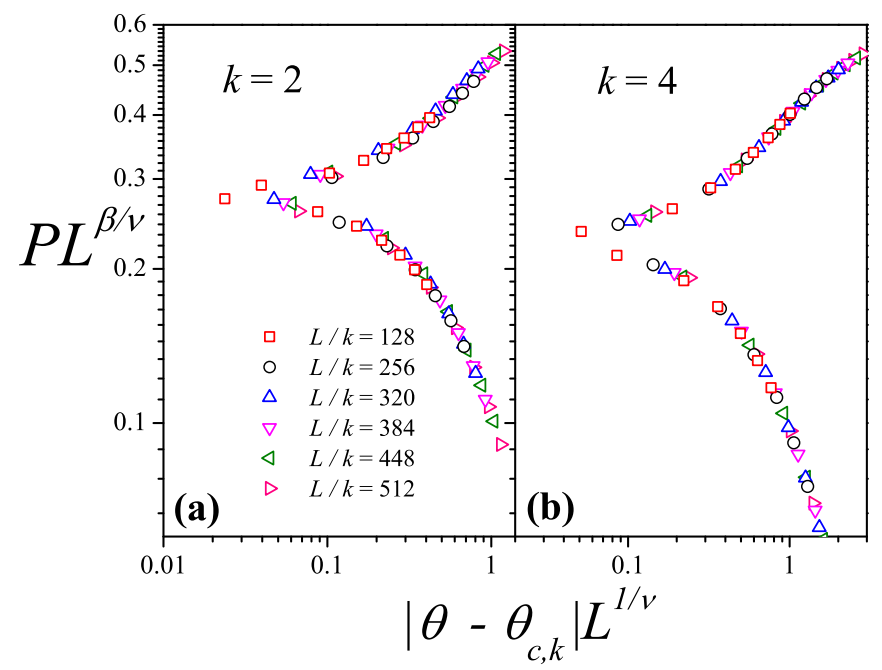

FIG. 10. Data collapsing of the percolation order parameter, $P L^{\beta / v}$ vs. $\left|\theta-\theta_{c, k}\right| L^{1 / v}$ for $k=2$ (a) and $k=4$ (b). The plots were made using $\theta_{c, k=2}=0.3619(2)$ and $\theta_{c, k=4}=0.2866(2)$ (see Fig. 6), and the exact $2 \mathrm{D}$ ordinary percolation exponents $v=4 / 3$ and $\beta=$ $5 / 36$.

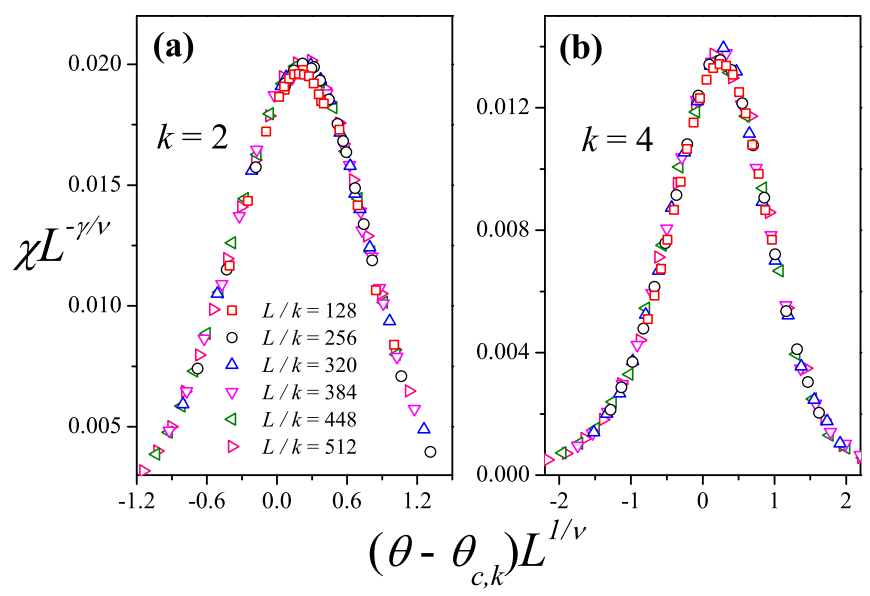

FIG. 11. Data collapsing of the susceptibility, $\chi L^{-\gamma / v}$ vs. $(\theta-$ $\left.\theta_{c, k}\right) L^{1 / v}$, for $k=2$ (a) and $k=4$ (b). The plots were made using $\theta_{c, k=2}=0.3619(2)$ and $\theta_{c, k=4}=0.2866(2)$ (see Fig. 6), and the exact $2 \mathrm{D}$ ordinary percolation exponents $v=4 / 3$ and $\gamma=43 / 18$.

value of the critical exponent of the $2 \mathrm{D}$ ordinary percolation, namely, $v=4 / 3$.

Once $v$ is known, the exponents $\gamma$ and $\beta$ can be determined from the scaling behavior of $\chi$ and $P$ at criticality [1],

$$
\chi=L^{\gamma / v} \bar{\chi}(u)
$$

and

$$
P=L^{-\beta / v} \bar{P}\left(u^{*}\right),
$$

where $u=\left(\theta-\theta_{c, k}\right) L^{1 / v}, u^{*}=\left|\theta-\theta_{c, k}\right| L^{1 / v}$, and $\bar{\chi}$ and $\bar{P}$ are the corresponding scaling functions. At the point where $\chi$ is maximal, $u=$ const and

$$
\chi_{\max } \propto L^{\gamma / v} .
$$

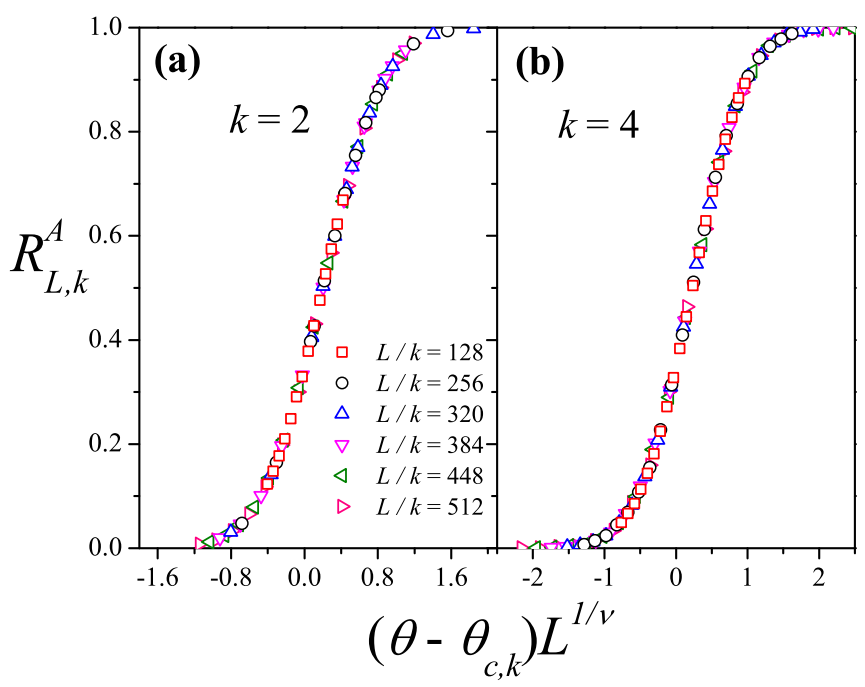

FIG. 12. Data collapsing of the percolation probability, $R_{L, k}^{A}(\theta)$ vs. $\left(\theta-\theta_{c, k}\right) L^{1 / v}$, for $k=2$ (a) and $k=4$ (b). The plots were made using $\theta_{c, k=2}=0.3619(2)$ and $\theta_{c, k=4}=0.2866(2)$ (see Fig. 6), and the exact 2D ordinary percolation exponent $v=4 / 3$. 


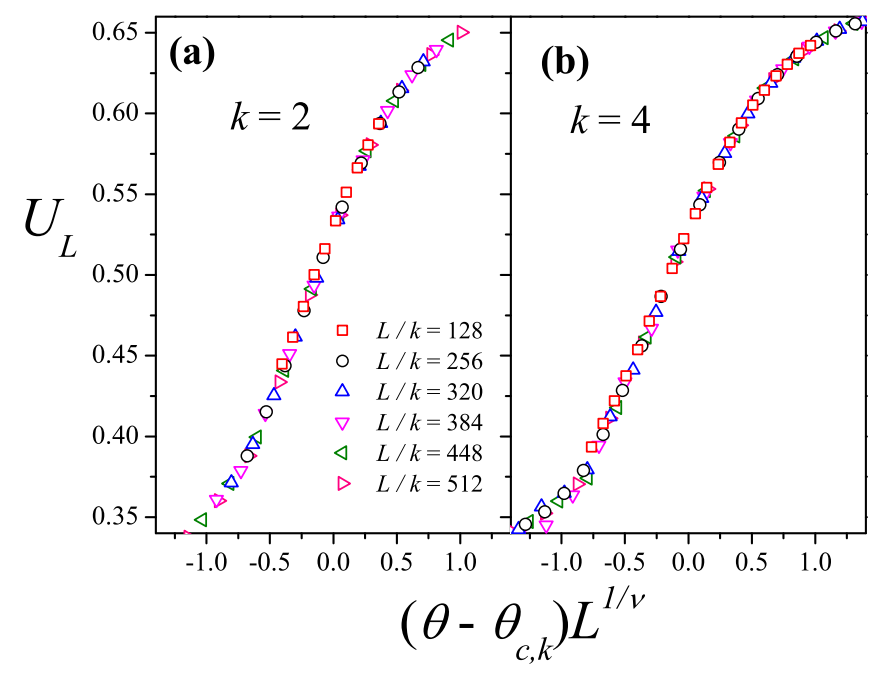

FIG. 13. Data collapsing of the the cumulant, $U_{L}$ vs. $(\theta-$ $\left.\theta_{c, k}\right) L^{1 / v}$ for $k=2$ (a) and $k=4$ (b). The plots were made using $\theta_{c, k=2}=0.3619(2)$ and $\theta_{c, k=4}=0.2866(2)$ (see Fig. 6), and the exact 2D ordinary percolation exponent $v=4 / 3$.

On the other hand, at the point where $d P / d \theta$ is maximal, $u^{*}=$ const and

$$
\left(\frac{d P}{d \theta}\right)_{\max }=L^{(-\beta / v+1 / v)} \bar{P}^{\prime}\left(u^{*}\right) \propto L^{(1-\beta) / v} .
$$

Then, the exponents $\gamma$ and $\beta$ can be determined by scaling the maximum values of the susceptibility [Eq. (20)] and the derivative of the order parameter [Eq. (21)], respectively. As an example of the application of Eqs. (20) and (21), the data for $\chi_{\max }$ and $(d P / d \theta)_{\max }$ are presented in Figs. 9(b) and 9 (c), respectively, for $k=2$ (circles) and $k=4$ (squares). The results obtained from the slopes of the curves agree very well with the exact values of $\gamma$ and $\beta$ for 2D ordinary percolation, $\gamma=43 / 18$ and $\beta=5 / 36$.

The scaling behavior has also been tested by plotting $P L^{\beta / v}$ versus $\left|\theta-\theta_{c, k}\right| L^{1 / v}, \chi L^{-\gamma / v}$ versus $\left(\theta-\theta_{c, k}\right) L^{1 / v}, R_{L}^{A}$ versus $\left(\theta-\theta_{c, k}\right) L^{1 / \nu}$, and $U$ versus $\left(\theta-\theta_{c, k}\right) L^{1 / \nu}$ and looking for data collapsing. Figures 10-13 show an excellent collapse of curves for two typical cases: $k=2$ and $k=4$. The plots were made using $\theta_{c, k=2}=0.3619(2)$ and $\theta_{c, k=4}=0.2866(2)$ (see Fig. 6), and the exact values of the critical exponents corresponding to $2 \mathrm{D}$ ordinary percolation $v=4 / 3, \gamma=43 / 18$, and $\beta=5 / 36$.

The study in Figs. 10-13 was repeated for other values of $k$. In all cases, the obtained results confirm that the percolation properties of the system presented here correspond to the same universality class as the $2 \mathrm{D}$ random percolation problem. Thus, although the increasing in the width of the deposited layer affects the value of the crossing points of the percolation probability functions, it does not alter the nature of the percolation transition occurring in the system.

\section{CONCLUSIONS}

In this paper, irreversible bilayer adsorption of straight semirigid rods (or linear $k$-mers) of different sizes on twodimensional square lattices has been studied. The adsorption kinetics was simulated by a random sequential adsorption algorithm, generalized to more than one deposited layer. In this scheme, (i) the linear $k$-mer is modeled as a chain composed of $k$ units, aligned along one of two lattice axes, and (ii) each $k$-mer unit can be adsorbed on either an empty or an occupied site in the first layer, resulting thus in the formation of a bilayer on the surface.

The filling process starts with an initially empty lattice and continues until the limiting state is reached and no more objects can be deposited due to the absence of empty site clusters of appropriate size and shape. Numerical simulations were performed for $k$-mer sizes ranging between 2 and 64 and $L \times L$ lattices with $L / k=64-640$. Then, for each coverage value, jamming and percolation properties have been investigated by finite-size scaling theory.

The behavior of the adsorption dynamics was monitored by following the cumulative frequency $\Phi_{L, k}(\theta)$ that a $L \times L$ lattice reaches a coverage $\theta$. The curves of $\Phi_{L, k}(\theta)$ for different lattice sizes cross at precise concentration, allowing an accurate determination of the jamming coverage $\theta_{j, k}$. The obtained results show that the dependence of $\theta_{j, k}$ on the particle size $k$ follows a decreasing law $(k \geqslant 8): \theta_{j, k}=0.7299(21)-$ $0.062(81) / k+3.54(55) / k^{2}$, with $\theta_{j, k \rightarrow \infty}=0.7299(21)$ the limit coverage for infinitely long $k$-mers. This value is lower than that observed for the one-dimensional bilayer problem $\theta_{j, k \rightarrow \infty}=0.7708$ (3) [43], indicating that the limiting coverage for infinitely long $k$-mers diminishes with lattice connectivity. A similar scenario has already been observed for the monolayer problem on 1D lattices [3], 2D square lattices $[10,49]$, and 3D simple cubic lattices [50].

In addition, by fitting $\Phi_{L, k}(\theta)\left[d \Phi_{L, k}(\theta) / d \theta\right]$ with the error [Gaussian] function, the maximum of the derivative of the cumulative frequency $\left[d \Phi_{L, k}(\theta) / d \theta\right]_{\max }$ and the width of the transition $\Delta_{L, k}$ are expected to behave asymptotically as $L^{1 / v_{j}}$, which allows to calculate the jamming exponent $v_{j}$. As predicted in Ref. [54], it was found that $\Delta_{L, k},\left(\frac{d \Phi_{L, k}}{d \theta}\right)_{\max } \propto M^{1 / 2}$, where $M$ is the number of elements (sites or nodes) that form the lattice. In this case, $M=2 L^{2}$ and accordingly, $v_{j}=1$. Finally, by using data collapse analysis, it was shown that the cumulative frequency behaves at criticality as $\Phi_{L, k}(\theta)=$ $\overline{\Phi_{k}}\left[\left(\theta-\theta_{j, k}\right) L^{1 / v_{j}}\right]$, where $\overline{\Phi_{k}}$ is the corresponding scaling function.

Once the limiting parameters $\theta_{j, k}$ were determined, the percolation properties of the system were studied. The percolation threshold exhibits a monotonic decreasing function when it is plotted as a function of the $k$-mer size: $\theta_{c, k}=A+$ $B \exp (-k / C)$, where $A=\theta_{c, k \rightarrow \infty}=0.0457(68)$ is the value of the percolation threshold by infinitely long $k$-mers, $B=$ $0.276(25)$, and $C=14(2)$. This behavior is completely different from that observed for the monolayer problem, where the percolation threshold shows a nonmonotonic $k$-mer size dependence: It decreases for small rod sizes, goes through a minimum around $k=13$, and finally increases slowly to reach a limit value $\theta_{c, k \rightarrow \infty}=0.615(1)[11-14]$.

The notorious differences between monolayer and bilayer problems were explained on the basis of the "cross-linking effect," by which some $k$-mers in the second layer are adsorbed on transversal rods previously deposited in the first layer. The presence of the cross-linking rule in the bilayer problem 
changes drastically the filling kinetics, strongly suppressing the formation of domains or islands of parallel $k$-mers. As established in the literature [11,13], it is precisely the formation of vertical and horizontal blocks of parallel $k$-mers which leads to the nonmonotonic behavior observed in the classical percolation problem of straight rigid $k$-mers on 2D square lattices.

The cross-linking effect also favors connectivity and, consequently, the percolation transition for the bilayer problem occurs at lower values of coverage than those corresponding to the monolayer problem. In addition, given a fixed value of $k$, percolation always occurs before jamming $\left(\theta_{c, k}<\theta_{j, k}\right)$, and the bilayer model presents percolation transition in all the $k$ space.

The results reported here complement those obtained for the monolayer problem [11-14], and could have potential application in the field of matrix composites filled with conductive elongated particles or fibers. Experimental studies in these systems have shown that the conductivity of the composites (or percolation threshold) decreases monotonically as the aspect ratio of the conductive particles is increased. The findings obtained in this paper demonstrate that the inclusion of the cross-linking rule in the model is essential to describe the monotonically decreasing of the percolation threshold curve. If the $k$-mers do not cross each other, such as occurs in the case of 2D discrete lattice models at monolayer, then a nonmonotonous behavior should be expected for the percolation threshold as a function of the size $k$. Thus, the theoretical predictions can guide future experiments investigating the effects of multilayer deposition and overlapping between the conductive rods on the shape of the curve of composite conductivity versus fiber size. This aspect represents a major challenge for experimentalists.

To conclude with the analysis of the percolation properties, the complete set of critical exponents $\nu, \beta$, and $\gamma$ was determined. The obtained results confirm that the percolation transition involved in the system belongs to the same universality class as the standard percolation problem. Even though the deposition mechanism, and consequent bilayer formation, drastically affect the behavior of the percolation threshold with $k$ and other critical properties (such as the crossing points of the percolation probability functions), it does not alter the nature of the percolation transition occurring in the system.

Future efforts will be devoted to extending the present analysis to $n$-layer systems with $n>2$.

\section{ACKNOWLEDGMENTS}

This work was supported in part by CONICET (Argentina) under Project No. PIP 112-201101-00615; Universidad Nacional de San Luis (Argentina) under Project No. 03-0816; and Ministry of Higher Education, Science and Technology (MESCYT-Dominican Republic) under Project No. FONDOCYT-2016-2017-084. The numerical work was done using the BACO parallel cluster [73] located at Instituto de Física Aplicada, Universidad Nacional de San Luis-CONICET, San Luis, Argentina. N.D.L.C.F. thanks the OAS Academic Scholarship Program (Graduate).
[1] D. Stauffer and A. Aharony, Introduction to Percolation Theory (Taylor \& Francis, London, 1994).

[2] J. W. Evans, Rev. Mod. Phys. 65, 1281 (1993).

[3] P. L. Krapivsky, S. Redner, and E. Ben-Naim, A Kinetic View of Statistical Physics (Cambridge University Press, Cambridge, UK, 2010).

[4] P. J. Flory, J. Am. Chem. Soc. 61, 1518 (1939).

[5] V. Privman, J. Adhesion 74, 421 (2000).

[6] A. Cadilhe, N. A. M. Araújo, and V. Privman, J. Phys.: Condens. Matter 19, 065124 (2007).

[7] J. J. Ramsden, Phys. Rev. Lett. 71, 295 (1993).

[8] P. R. Van Tassel, L. Guemouri, J. J. Ramsden, G. Tarjus, P. Viot, and J. Talbot, J. Colloid Interface Sci. 207, 317 (1998).

[9] M. Rabe, D. Verdes, and S. Seeger, Adv. Colloid Interface Sci. 162, 87 (2011).

[10] B. Bonnier, M. Hontebeyrie, Y. Leroyer, C. Meyers, and E. Pommiers, Phys. Rev. E 49, 305 (1994).

[11] Y. Leroyer and E. Pommiers, Phys. Rev. B 50, 2795 (1994).

[12] G. Kondrat and A. Pȩkalski, Phys. Rev. E 63, 051108 (2001).

[13] Y. Y. Tarasevich, N. I. Lebovka, and V. V. Laptev, Phys. Rev. E 86, 061116 (2012).

[14] M. G. Slutskii, L. Y. Barash, and Y. Y. Tarasevich, Phys. Rev. E 98, 062130 (2018).

[15] G. Kondrat, Z. Koza, and P. Brzeski, Phys. Rev. E 96, 022154 (2017).

[16] V. Cornette, A. J. Ramirez-Pastor, and F. Nieto, Eur. Phys. J. B 36, 391 (2003).
[17] M. Pawłowska, S. Żerko, and A. Sikorski, J. Chem. Phys. 136, 046101 (2012).

[18] M. Pawłowska and A. Sikorski, J. Mol. Model. 19, 4251 (2013).

[19] P. Adamczyk, P. Romiszowski, and A. Sikorski, J. Chem. Phys. 128, 154911 (2008).

[20] M. Nakamura, Phys. Rev. A 36, 2384 (1987).

[21] K. Shida, R. Sahara, M. N. Tripathi, H. Mizuseki, and Y. Kawazoe, Mater. Trans. 51, 1141 (2010).

[22] I. A. Kriuchevskyi, L. A. Bulavin, Y. Y. Tarasevich, and N. I. Lebovka, Condens. Matter Phys. 17, 33006 (2014).

[23] A. J. Ramirez-Pastor, P. M. Centres, E. E. Vogel, and J. F. Valdés, Phys. Rev. E 99, 042131 (2019).

[24] A. P. Chatterjee, J. Chem. Phys. 139, 224904 (2013).

[25] H. Meyer, P. van der Schoot, and T. Schilling, J. Chem. Phys. 143, 044901 (2015).

[26] S. P. Finner, M. I. Kotsev, M. A. Miller, and P. van der Schoot, J. Chem. Phys. 148, 034903 (2018).

[27] T. Drwenski, S. Dussi, M. Dijkstra, R. van Roij, and P. van der Schoot, J. Chem. Phys. 147, 224904 (2017).

[28] T. Drwenski, R. van Roij, and P. van der Schoot, J. Chem. Phys. 149, 054902 (2018).

[29] S. I. White, R. M. Mutiso, P. M. Vora, D. Jahnke, S. Hsu, J. M. Kikkawa, J. Li, J. E. Fischer, and K. I. Winey, Adv. Funct. Mater. 20, 2709 (2010).

[30] R. M. Mutiso, M. C. Sherrott, J. Li, and K. I. Winey, Phys. Rev. B 86, 214306 (2012). 
[31] A. Golovnev and M. E. Suss, J. Chem. Phys. 149, 144904 (2018).

[32] S. P. Finner, I. Pihlajamaa, and P. van der Schoot, J. Chem. Phys. 152, 064902 (2020).

[33] L. Fingold and J. T. Donnell, Nature 278, 443 (1979).

[34] Z. Adamczyk, T. Babros, J. Czarnecki, and T. G. M. van de Ven, Adv. Colloid Interface Sci. 19, 183 (1983).

[35] V. Privman, H. L. Frisch, N. Ryde, and E. Matijveć, J. Chem. Soc., Faraday Trans. 87, 1371 (1991).

[36] L. Chen, R. S. Smith, B. D. Kay, and Z. Dohnálek, Surf. Sci. 650, 83 (2016).

[37] T. H. Nguyen, V. Q. Nguyen, A. T. Duong, and S. Cho, J. Alloys Compd. 810, 151968 (2019).

[38] A. J. Groszek, Proc. R. Soc. Lond. Ser. A 314, 473 (1970).

[39] M. C. Bartelt and V. Privman, J. Chem. Phys. 93, 6820 (1990).

[40] P. Nielaba and V. Privman, Phys. Rev. A 45, 6099 (1992).

[41] V. Privman and J.-S. Wang, Phys. Rev. A 45, R2155 (1992).

[42] P. Nielaba, V. Privman, and J.-S. Wang, Berich. Bunsen. Gesell. 98, 451 (1994).

[43] N. De La Cruz Félix, P. M. Centres, A. J. Ramirez-Pastor, E. E. Vogel, and J. F. Valdés, Phys. Rev. E 102, 012106 (2020).

[44] R. H. Swendsen, Phys. Rev. A 24, 504 (1981).

[45] E. Ben-Naim and P. L. Krapivsky, J. Phys. A 27, 3575 (1994).

[46] N. O. Wolf, J. W. Evans, and D. K. Hoffman, J. Math. Phys. (NY) 25, 2519 (1984).

[47] A. Rényi, Sel. Transl. Math. Stat. Probab. 4, 203 (1963); Magyar Tud. Akad. Mat. Kutató Int. Közl. 3, 109 (1958).

[48] E. J. Perino, D. A. Matoz-Fernandez, P. M. Pasinetti, and A. J. Ramirez-Pastor, J. Stat. Mech. (2017) 073206.

[49] M. Dolz, F. Nieto, and A. J. Ramirez-Pastor, Phys. Rev. E 72, 066129 (2005).

[50] G. D. García, F. O. Sanchez-Varretti, P. M. Centres, and A. J. Ramirez-Pastor, Physica A 436, 558 (2015).

[51] M. E. J. Newman and R. M. Ziff, Phys. Rev. E 64, 016706 (2001).

[52] N. Vandewalle, S. Galam, and M. Kramer, Eur. Phys. J. B 14, 407 (2000).

[53] L. S. Ramirez, P. M. Centres, and A. J. Ramirez-Pastor, Phys. Rev. E 100, 032105 (2019).
[54] P. M. Pasinetti, L. S. Ramirez, P. M. Centres, A. J. RamirezPastor, and G. A. Cwilich, Phys. Rev. E 100, 052114 (2019).

[55] F. Yonezawa, S. Sakamoto, and M. Hori, Phys. Rev. B 40, 636 (1989).

[56] J. Hoshen and R. Kopelman, Phys. Rev. B 14, 3438 (1976).

[57] K. Binder, Rep. Prog. Phys. 60, 487 (1997).

[58] S. Biswas, A. Kundu, and A. K. Chandra, Phys. Rev. E 83, 021109 (2011).

[59] A. K. Chandra, Phys. Rev. E 85, 021149 (2012).

[60] J. Cardy, J. Phys. A: Math. Gen. 25, L201 (1992).

[61] J. J. H. Simmons, P. Kleban, and R. M. Ziff, J. Phys. A: Math. Gen. 40, F771 (2007).

[62] G. M. T. Watts, J. Phys. A: Math. Gen. 29, L363 (1996).

[63] W. Selke and L. N. Shchur, J. Phys. A 38, L739 (2005).

[64] W. Selke, J. Stat. Mech.: Theory Exp. (2007) P04008.

[65] G. D. Garcia, F. O. Sanchez-Varretti, P. M. Centres, and A. J. Ramirez-Pastor, Eur. Phys. J. B 86, 403 (2013).

[66] We call this effect a cross-linking effect due the fact that a $k$-mer adsorbed along the $x[y]$ direction, with one of its units located in a given position $(i, j, 1[2])$ is connected (or linked) to a perpendicular $k$-mer deposited along the $y[x]$ direction, with one of its units located in the position $(i, j, 2[1])$. The link occurs through the formation of a pair of nearest-neighbor occupied sites in the positions $(i, j, 1)$ and $(i, j, 2)$.

[67] C. Brosseau, A. Beroual, and A. Boudida, J. Appl. Phys. 88, 7278 (2000).

[68] F. Du, J. E. Fischer, and K. I. Winey, J. Polym. Sci. B 41, 3333 (2003).

[69] J. L. Mietta, R. M. Negri, and P. I. Tamborenea, J. Phys. Chem. C 118, 20594 (2014); and references therein.

[70] N. Ohtake, T. Kuzumaki, and Y. Mitsuda, High Polymer 52, 888 (2003).

[71] S. A. Gordeyev, F. J. Macedo, J. A. Ferreira, F. W. J. van Hattum, and C. A. Bernardo, Physica B 279, 33 (2000).

[72] T. Natsuki, M. Endo, and T. Takahashi, Physica A 352, 498 (2005).

[73] Available at http://cluster_infap.unsl.edu.ar/wordpress. 